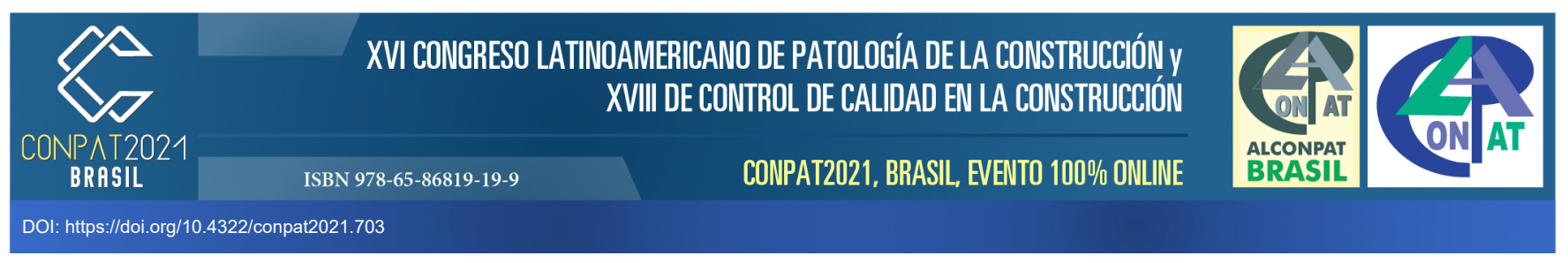

\title{
Comportamiento a fatiga de morteros que incluyen residuos industriales derivados del poliuretano
}

\author{
V. Calderón*, A. Alonso, R: Arroyo, L. Alameda, S. González-Moreno. S. Gutiérrez-González \\ *Autora de Contacto: vcalderon@ubu.es \\ ${ }^{1}$ Departamento de Construcciones Arquitectónicas e I.C.T, Escuela Politécnica Superior, \\ Universidad de Burgos (España)
}

\begin{abstract}
RESUMEN
El objetivo del trabajo consiste en analizar el comportamiento a la fatiga de lo morteros de cemento que incluyen residuos industriales derivados del poliuretano.

La metodología se basa en la fabricación de eco morteros aligerados mediante la sustitución de residuo en proporciones volumétricas del $50 \%, 60 \%$ y $75 \%$, aplicando ciclos de carga y descarga a compresión una vez calculado el módulo de elasticidad estático inicial en cada una de las dosificaciones. Aunque existen estudios previos con ensayos de la de la misma naturaleza, son escasos o inexistentes los que se aplican a este tipo de materiales eco sostenibles ligeros.

El buen comportamiento a fatiga se corrobora a través del análisis de la microestructura mediante tomografía axial computarizada, no observando fracturas significativas comparando secciones antes y después de los ciclos de carga, lo que indica que no se produce el colapso de las estructuras.
\end{abstract}

Palabras clave: Mortero de poliuretano; módulo de elasticidad; durabilidad; comportamiento frente a fatiga. 


\section{INTRODUCCIÓN}

El desarrollo sostenible y el respeto por el medio ambiente son valores sociales que hay que fomentar mediante la puesta en práctica de estrategias que valoricen los residuos generados en los procesos industriales y los transformen en recursos. Es lo que se conoce como "economía circular" y es la apuesta de la sociedad por encontrar solución a los problemas medioambientales que genera nuestro modelo de desarrollo.

La utilización de residuos industriales en la fabricación de materiales de construcción es el fundamento de múltiples investigaciones que buscan poner en valor los residuos, transformándolos en materia prima sustitutiva de los recursos naturales, evitando así los costes de gestión para su procesado o los costes medioambientales para su depósito en vertedero.

El uso de residuos poliméricos en la fabricación de morteros y hormigones supone un importante beneficio medioambiental. El uso de este tipo de morteros tiene las ventajas añadidas de reducir las cargas sobre las estructuras, abaratar el coste del transporte y mejorar las propiedades de aislamiento térmico.

No obstante, en la normativa europea, dentro de los ensayos obligados de durabilidad, no se incluye el comportamiento de morteros cuando trabajan bajo cargas variables, al no ser materiales puramente estructurales. Sin embargo, cuando forman parte de muros de carga o de tabiques de edificios, tienen que soportar la acción combinada de esfuerzos de flexión y compresión, producidos por variaciones térmicas, tensiones de humedad-sequedad, porque se ven afectados por la interacción de los elementos de los que forman parte o por la propia rigidez de estos elementos que impiden su movimiento, dando lugar a microfisuras en la matriz del mortero que derivan en la rotura por efecto de la fatiga, y que, a medio plazo, pueden producir el colapso de la unidad constructiva. Tal es el caso de los morteros de albañilería colocados en obra en revestimientos horizontales y verticales y en juntas en fábricas de ladrillo, piedra, o bloque de hormigón.

Existen muy pocos estudios relativos al comportamiento inelástico de los materiales poliméricos, y prácticamente ningún trabajo de investigación en lo referente a los efectos de la fatiga en morteros de cemento con polímeros. Ello ha llevado a realizar esta investigación que pretende mostrar que el comportamiento frente a la fatiga de los morteros de cemento aligerados con residuos industriales de poliuretano, es similar al de los morteros de referencia usados comúnmente en trabajos de albañilería. Este estudio pretende conocer el comportamiento a fatiga y las deformaciones de los morteros aligerados con PUR, en comparación con los morteros comunes de albañilería, para confirmar la aptitud de estos morteros ligeros.

\section{PROCEDIMIENTO}

La metodología empleada para la realización de los ensayos de fatiga en morteros, difiere sensiblemente de unos autores a otros, tanto para los intervalos de carga empleada, como para la frecuencia de los ciclos y su número. Así, una carga máxima por ciclo del 70\%-80\% de la carga de rotura supone una carga muy elevada para ciertos autores (A. Carpinteri et.al, K. Sobhan et.al.), que se puede compensar con un número pequeño de ciclos.

Tratándose de hormigón, distintos autores emplean diversas metodologías, estudiando el comportamiento del material desde los 20 ciclos hasta los 2.000.000 de ciclos, con ratios de carga 
que oscilan desde 0,5 a 0,9 sobre la carga unitaria de rotura y frecuencias de 2 a $10 \mathrm{~Hz}$ (J. Zhou et al., K. Dyduch et al.)

\subsection{Materias primas}

\subsubsection{Cemento.}

Se han utilizado dos tipos de cemento comercial normalizado, CEM II, con una resistencia media $32,5 \mathrm{MPa}$ a los 28 días, de acuerdo con lo especificado en la norma EN 197-1.

\subsubsection{Arena}

El árido empleado en las mezclas es una arena silícea de origen sedimentario extraída directamente de la cantera, secada previamente y sin ningún otro tratamiento posterior a su recepción en el laboratorio. La caracterización de la arena se ha realizado de acuerdo con las especificaciones de la norma europea EN 13139 con un tamaño máximo del árido de 4mm.

\subsubsection{Residuo industrial derivado de $P U$}

Los residuos de poliuretano, que vienen en forma de trozos de panel o sin forma, se trituran previamente para obtener partículas de tamaño máximo similar al del árido, hasta tamaño de partícula de $4 \mathrm{~mm}$. El residuo procede de la industria de componentes del automóvil con una densidad aparente de $59 \mathrm{~kg} / \mathrm{m}^{3}$ y densidad real de $1209 \mathrm{~kg} / \mathrm{m}^{3}$ después de triturado.

\subsection{Diseño de las mezclas}

Para el proceso experimental se han fabricado morteros de referencia de cemento, arena y agua, con relación cemento/arena en peso 1/4.

También se han elaborado los morteros en los que se ha sustituido un porcentaje del 50\%, 60\% o $75 \%$ del volumen de arena, por residuo, con la dosificación detallada en la Tabla 1.

\begin{tabular}{lcccc}
\hline \multicolumn{1}{c}{ Tabla 1. } & \multicolumn{3}{c}{ Cantidad } \\
\hline Designación & $\begin{array}{c}\text { Sustitución } \\
\text { de cemento } \\
\text { kg/m }\end{array}$ & $\begin{array}{c}\text { volumen de arena } \\
\text { por residuo }\end{array}$ & $\begin{array}{c}\text { Tensión mín-máx } \\
\text { s/rotura }\end{array}$ & $\begin{array}{c}\mathbf{N}^{\mathbf{3}} \\
\text { ciclos/Frecuencia }\end{array}$ \\
\hline MR & 356 & -- & $20 \%-60 \%$ & $300.000 / 2 \mathrm{~Hz}$ \\
\hline MPU50 & 467 & 50 & $20 \%-60 \%$ & $300.000 / 2 \mathrm{~Hz}$ \\
MPU60 & 488 & 60 & $20 \%-60 \%$ & $300.000 / 2 \mathrm{~Hz}$ \\
MPU75 & 343 & 75 & $20 \%-60 \%$ & $300.000 / 2 \mathrm{~Hz}$ \\
\hline
\end{tabular}


La cantidad de agua de los morteros se ha fijado en todos los casos para conseguir una consistencia adecuada para su correcta puesta en obra, (consistencia plástica), ensayada de acuerdo con la norma EN 1015-3.

Se han fabricado las mezclas en amasadora mecánica de acuerdo con el procedimiento operatorio descrito en la norma EN 196-1 y se han moldeado 4 series de tres probetas cada una para cada tipo de mezcla, de dimensiones $160 \mathrm{~mm}$ x $40 \mathrm{~mm}$ x $40 \mathrm{~mm}$, que es el tamaño de probeta para realizar los ensayos de flexión y compresión, de acuerdo con la norma EN 1015-11. Posteriormente se han desmoldado y se han curado en la cámara húmeda durante 28 días.

\subsection{Procedimiento experimental}

Para calcular las resistencias mecánicas de los morteros se han seleccionado 2 probetas de cada serie, tanto de los morteros con espumas como de los morteros de referencia. En primer lugar, se ha determinado la resistencia a flexión y compresión a los 7 y 28 días, obteniendo unos resultados medios de tensión en rotura, a 28 días, que servirán como referencia para la posterior aplicación de las cargas empleadas en los ensayos de fatiga.

Ha sido necesario determinar el Módulo de Elasticidad Estática bajo Compresión, a través de ensayos directos, porque en general las formulaciones matemáticas para calcular el módulo de elasticidad no suelen tener en cuenta la composición de los agregados (F. Duplan et al.). Para el cálculo del Módulo de Elasticidad Estático se han moldeado probetas de dimensiones $120 \mathrm{~mm} \mathrm{x}$ $50 \mathrm{~mm}$ x $50 \mathrm{~mm}$, que es el tamaño de probeta para realizar los ensayos de módulo de elasticidad estático, de acuerdo con la norma EN 14580.

A cada una de las probetas de cada tipo de mortero, se les han colocado galgas extensiométricas de $60 \mathrm{~mm}$ de longitud en dos de sus caras opuestas y se les ha sometido a ciclos de carga y descarga. Los ensayos de fatiga se han concretado en dos fases. La primera fase ha consistido en la aplicación de 300.000 ciclos de carga y descarga a compresión sobre una superficie de $40 \mathrm{~mm}$ x $40 \mathrm{~mm}$ con una variación de la tensión entre el $20 \%$ y el $60 \%$ de la tensión de rotura, para cada tipo de mortero, y una frecuencia de $2 \mathrm{~Hz}$. Esta frecuencia es suficiente para medir la resistencia a la fatiga sin necesidad de realizar un elevado número de ciclos. (N. Oneschkow) Además, la frecuencia de $2 \mathrm{~Hz}$ es muy superior a las frecuencias que pueden dar lugar a ciclos de vida muy cortos por acumulación de deformaciones plásticas del polímero.

Se han registrado los datos de deformación de las probetas durante 10 segundos (20 ciclos), cada hora, con 5 mediciones de la deformación de la probeta en cada ciclo, con una carga total de 50 $\mathrm{kN}$.

\subsection{Tomografía axial computarizada}

Se ha realizado una tomografía axial computarizada para visualizar, de manera no invasiva, la microestructura de los moteros antes y después de ser sometidos al ensayo de fatiga. 


\section{RESULTADOS}

Los resultados de la tensión de rotura a flexión y compresión de estos morteros ligeros indican una disminución de resistencias de los morteros con agregados de residuos de poliuretano en función de la cantidad de residuo dosificado en cada una de las mezclas ensayadas. El módulo de elasticidad estática a compresión se ha obtenido calculando los valores de las tensiones y deformaciones en los intervalos que señala la norma UNE-EN 1352. Los resultados de los módulos de elasticidad y las resistencias a compresión antes y después de realizados los ensayos se indican en la Tabla 2.

Tabla 2.

\begin{tabular}{lccc}
\hline Designación & $\begin{array}{c}\text { Módulo de elasticidad } \\
{\left[\mathbf{N} / \mathbf{m m}^{2}\right]}\end{array}$ & $\begin{array}{c}\text { Tensión rotura compresión } \\
\text { antes del ensayo } \\
{[\mathbf{M P a}]}\end{array}$ & $\begin{array}{c}\text { Tensión rotura compresión } \\
\text { después del ensayo } \\
{[\mathbf{M P a}]}\end{array}$ \\
\hline MR & 15.758 & 11.62 & 11.49 \\
\hline MPU50 & 8.926 & 10.05 & 10.31 \\
MPU60 & 3.684 & 4.58 & 3.50 \\
MPU75 & 1.642 & 1.81 & 2.44 \\
\hline
\end{tabular}

Los resultados obtenidos muestran la lógica relación entre la resistencia de rotura a compresión y los módulos de elasticidad, siendo los módulos menores en función de la menor resistencia a compresión de los distintos tipos de mortero, y que coincide básicamente con el contenido de residuo aligerante, pudiéndose llegar a establecer una relación no lineal entre las distintas cantidades de contenido de residuo y el módulo de elasticidad.

En todos los casos, además de la lógica disminución de las densidades aparentes, en función del contenido de residuo de poliuretano, se puede observar una pérdida de resistencias, poco significativa en los morteros con sustitución del $50 \%$ de arena por residuo, pero más importante en los morteros con sustitución del $60 \%$ y $75 \%$. Sin embargo, en estos últimos morteros, que se pueden considerar como morteros ligeros (densidad inferior a $1300 \mathrm{~kg} / \mathrm{m}^{3}$ ), las resistencias a compresión obtenidas a los 28 días permiten su utilización como morteros para revestimientos continuos (revoco y enlucido) y para morteros de albañilería (sentado de fábricas).

Los resultados de deformación máxima, deformación residual y la amplitud de deformación a 300.00 ciclos se detallan los módulos de elasticidad y las resistencias a compresión antes y después de realizados los ensayos se indican en la Tabla 3.

Tabla 3. Deformaciones de los morteros

\begin{tabular}{lcccc}
\hline Designación & \multicolumn{2}{c}{$\begin{array}{c}\text { Deformación máxima } \\
(\mathbf{m m} / \mathbf{m})\end{array}$} & $\begin{array}{c}\text { Deformación residual 60 } \\
\text { minutos sin carga. } \\
(\mathbf{m m} / \mathbf{m})\end{array}$ & $\begin{array}{c}\text { Amplitud deformación } \\
\mathbf{3 0 0 . 0 0 0} \text { ciclos. } \\
\mathbf{( m m} / \mathbf{m})\end{array}$ \\
\cline { 2 - 3 } MR & $\mathbf{1 2 0}$ ciclos & $\mathbf{3 0 0 . 0 0 0}$ ciclos & & 0,5067 \\
\hline MPU50 & 0,8532 & 1,0530 & 0,5245 & 0,1911 \\
\hline MPU60 & 1,1924 & 1,5138 & 0,8783 & 0,3150 \\
\hline MPU75 & 1,6550 & 2,5329 & 0,6387 \\
\hline
\end{tabular}


Table 4. Deformaciones de los morteros en el ensayo de fatiga.

\begin{tabular}{lcccc}
\hline \multirow{2}{*}{ Designación } & \multicolumn{2}{c}{$\begin{array}{c}\text { Deformación máxima } \\
(\mathbf{m m} / \mathbf{m})\end{array}$} & $\begin{array}{c}\text { Deformación residual 60 } \\
\text { minutos sin carga. } \\
(\mathbf{m m} / \mathbf{m})\end{array}$ & $\begin{array}{c}\text { Amplitud deformación } \\
\mathbf{3 0 0 . 0 0 0} \text { ciclos. } \\
\mathbf{( m m} / \mathbf{m})\end{array}$ \\
\cline { 2 - 3 } MR & $\mathbf{1 2 0}$ ciclos & $\mathbf{3 0 0 . 0 0 0}$ ciclos & & 0,5067 \\
\hline MPU50 & 0,8532 & 1,0530 & 0,5245 & 0,1911 \\
\hline MPU60 & 1,1924 & 1,5138 & 0,8783 & 0,3150 \\
\hline MPU75 & 1,6550 & 2,5329 & 1,1745 & 0,6387 \\
\hline
\end{tabular}

Una vez concluidos los 300.000 ciclos con carga del $20 \%$ al $60 \%$ de la carga de rotura, los resultados de resistencia a rotura de los morteros prácticamente coinciden con los obtenidos antes de ser sometidos al ensayo. En todo caso se observa un ligero aumento de la resistencia obtenida, con respecto a la registrada a la edad de 28 días.

Finalmente, la Figura 1 muestra la reconstrucción de las imágenes en 3D de la macroporosidad (tamaños de poros $>170 \mu \mathrm{m}$ ) según la relación de arena reemplazada por las partículas de polímero. Las imágenes muestran una gran heterogeneidad, tanto en la distribución como en el tamaño de los poros que puede dar lugar a fracturas más importantes en las primeras etapas de tensión, con deformaciones plásticas. Se ha realizado la comparación entre las imágenes de los morteros tomografiados, antes y después de ser sometidos al ensayo de fatiga. En ambos casos se puede apreciar un importante estado de fisuración en las probetas sometidas al ensayo de fatiga, estado que se hace más evidente en los morteros de referencia.

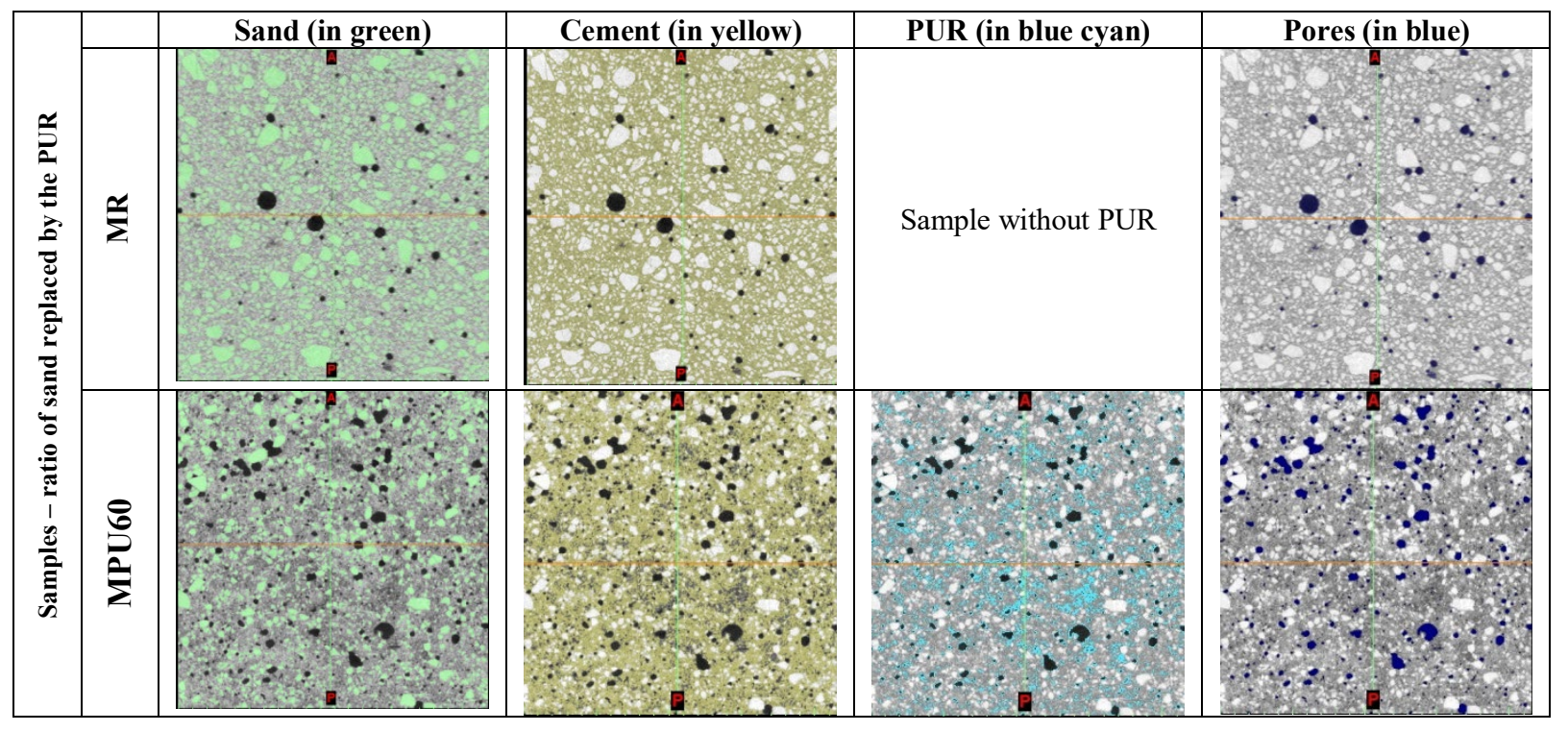




\section{CONCLUSIONES}

Los resultados de los ensayos de resistencia frente a la fatiga de los morteros de cemento, con sustitución parcial de arena por residuo triturado de espuma rígida de poliuretano, muestran que el comportamiento elástico, en ciclos repetitivos de carga y descarga a compresión, es similar al de los morteros de referencia cuando la carga aplicada no supera el $60 \%$ de la carga de rotura a compresión. La amplitud de la deformación, así como las deformaciones máximas y residuales, son mayores en función del mayor contenido de residuo. Esta mayor deformación admisible hace que, en caso de deformación obligada, por deformación del soporte del que forman parte, las mezclas con residuo de poliuretano se adapten mejor a deformaciones impuestas, pues admiten un mayor rango de deformación.

La tensión de rotura a compresión después de 300.000 ciclos, con carga variable del $20 \%$ al $60 \%$ de la de rotura, es similar a la obtenida antes de la realización del ensayo, tanto en los morteros de referencia como en los morteros ligeros. El mortero con contenido del $75 \%$ de espuma presenta un comportamiento muy diferente al resto de morteros, con deformaciones excesivas en los primeros ciclos y deformaciones residuales importantes.

La tomografía axial computarizada (TAC) no muestra fracturas significativas en las secciones antes y después del ensayo de fatiga, lo que indica que no se ha producido el colapso de las muestras.

\section{AGRADECIMIENTOS}

Este estudio se ha llevado a cabo gracias al apoyo económico del proyecto BU070P20 financiado por el Fondo Europeo de Desarrollo Regional (FEDER) y la Junta de Castilla y León (España).

\section{REFERENCIAS}

[1] A. Carpinteri, M. Corrado, G. Lacidogna, 2012. Three different approaches for damage domain characterization in disordered materials: fractal energy density, value statistics, renormalization group theory, Mech. Mater. 53, 15-28.

[2] K. Sobhan, L. González, D.V. Reddy, 2016. Durability of a pavement foundation made from recycled aggregate concrete subjected to cyclic wet-dry exposure and fatigue loading, Mater. Struct. 49, 2271-2284.

[3] J. Zhou, M. Zeng, Q. Wang, J. Yang, T. Lin, 2016. Flexural fatigue behavior of polymer modified previous concrete with single sized aggregates, Constr. Build. Mater. 124, 897-905.

[4] K. Dyduch, M. Szerszen, 1994. Experimental investigation of the fatigue strength of plain concrete under high compressive loading, Mater. Struct. 27, 505-509.

[5] F. Duplan, A. Abou-Chakra, A. Turatsinze, G. Escadeillas, S. Brule, F. Masse, 2014. Prediction of modulus of elasticity based on micromechanics theory and application to lowstrength mortars, Constr. Build. Mater. 50, 437-447.

[6] N. Oneschkow, 2016. Fatigue behavior of high-strength concrete with respect to strain and stiffness, Int. J. Fatigue 87, 38-49. 